Series in

Modern

Condensed

Matter

Physics

Vol. 2

\title{
QUANTUM DISSIPATIVE SYSTEMS
}

\author{
Ulrich Weiss \\ Institute for Theoretical Physics \\ University of Stuttgart
}




\section{Published by}

World Scientific Publishing Co. Pte. Ltd.

P O Box 128, Farrer Road, Singapore 912805

USA office: Suite 1B, 1060 Main Street, River Edge, NJ 07661

UK office: 57 Sheiton Street, Covent Garden, London WC2H 9HE

\section{British Llbrary Cataloguing-in-Publication Data \\ A catalogue record for this book is available from the British Library.}

First published 1993

First reprint 1998

\section{Series In Modern Condensed Matter Physics Vol. 2: QUANTUM DISSIPATIVE SYSTEMS}

Copyright 1993 by World Scientific Publishing Co. Pte. Ltd.

All rights reserved. This book, or parts thereof, may not be reproduced in any form or by any means, electronic or mechanical, including photocopying, recording or any information storage and retrieval system now known or to be invented, without written permission from the Publisher.

For photocopying of material in this volume, please pay a copying fee through the Copyright Clearance Center, Inc., 222 Rosewood Drive, Danvers, MA 01923, USA. In this case permission to photocopy is not required from the publisher.

ISBN 981-02-0754-9

ISBN 981-02-0755-7 (pbk)

Printed in Singapore. 
This page is intentionally left blank 


\section{Preface}

This book is an outgrowth of a series of lectures which I taught at the ICTP at Trieste and at the University of Stuttgart during the spring and summer of 1991. The purpose of my lectures was to present the approaches and techniques that accurately treat quantum processes in the presence of frictional influences.

The problem of open quantum systems has been around since the beginnings of quantum mechanics. Important contributions to this general area have been made by researchers working in fields as diverse as solid-state physics, chemical physics, biophysics, quantum measurement theory, quantum optics, nuclear and particle physics. Often, there has been used, and still is used, a language well known in one context or one field, yet sufficiently different from others that it is not altogether easy to make out the connection. Here, I offer a collection of ideas and examples rather than a comprehensive review of the topic and the history.

The central theme is the space-time functional integral or path integral formulation of quantum theory. This approach is particularly well suited for treating the quantum generalization of friction. Here we are faced to understand the behavior of a system with few quantal degrees of freedom coupled to a thermal reservoir. After integrating out the bath while keeping the system's coordinates fixed we get the influence functional describing the influence of the many bath degrees of freedom on the few relevant ones. This leads to an effective action weighting the paths of the open system in the functional integral description. Indeed, if one wishes to perform numerical computations on a rigorous level, there are no alternatives to this approach at present.

Path integration in condensed matter and chemical physics has become a growth industry in the last one or two decades. A newcomer to this thriving field may not yet be very familiar with the path integral method. Here, I do assume a knowledge of standard text book quantum mechanics and statistical mechanics augmented by a knowledge of Feynman's approach on a first introductory level. The books by Baym [1], Chandler [2], Feynman and Hibbs [3], and by Feynman [4] provide the elementary material in this regard. Further background and supplementary material on the path integral method are contained in the books by Schulman [5] and by Kleinert [6]. However, advanced mastery of these subjects is not necessary.

Some of the more sophisticated concepts, such as preparation functions, propagating functions, and correlation functions, are basic to the development as it is presented here. To cover this material at an introductory level, I make frequent use of simplified models. In this way, I can keep the mathematics relatively simple.

Many of the problems, methods, and ideas which are discussed here have become essential to the current understanding of quantum statistical mechanics. I 
have made a considerable effort to make the material largely self-contained. Thus, although the theoretical tools are not developed systematically and in its full beauty, the material may be useful to many graduate students to become familiar with the field and learn the methods. For the most part, I refrain from just quoting results without explaining where they come from. With regard to citations, I have tried to give references to the historical development and also to provide a selection of the very recent important ones. But the list is surely not a comprehensive bibliography.

This book exists because of the physics I learned and enjoyed from the fertile collaboration with Hermann Grabert, Peter Hänggi, Gert-Ludwig Ingold, Peter Riseborough, and Maura Sassetti. I am particularly indebted to Maura who took time off her research to weed out points of confusion and who persistently encouraged me to finish this venture. I am also grateful to my students Reinhold Egger, Manfried Milch, Jürgen Stockburger, and Dietmar Weinmann for helpful comments concerning the presentation of many subjects discussed here and for preparing the figures.

In writing this book, I have benefited from the discussion with many companions; in particular Uli Eckern, Enrico Galleani d'Agliano, Anthony J. Leggett, Hajo Leschke, Franco Napoli, Albert Schmid, Gerd Schön, Larry Schulman, Peter Talkner, Valerio Tognetti, Andrei Zaikin, and Wilhelm Zwerger, who helped me in increasing my understanding of many of the subjects which are discussed here.

It is a pleasure to thank my teacher, colleague, and friend Wolfgang Weidlich for many fruitful discussions over the years.

Finally, and most importantly, I am deeply grateful to my wife Christel and my children Ulrike, Jan, and Meike for their infinite patience and omnipresent sympathy.

\section{Stuttgart}

October 1992

Ulrich Weiss 


\section{Contents}

1 Introduction $\quad 1$

I GENERAL THEORY 3

2 Traditional approaches to open quantum systems 3

2.1 Classical Langevin equation ................... . 3

2.2 New schemes of quantization ................. 4

2.3 System-plus-reservoir methods ................ 5

2.3.1 Generalized master equations ... . . . . . . . . . . 5

2.3.2 Generalized q-number Langevin equations . . . . . . . . . . 7

2.3.3 Quantum and quasiclassical Langevin equation ....... 8

2.3.4 Phenomenological methods ................ 10

3 System-plus-reservoir models $\quad 10$

3.1 Thermal reservoir with linear response . . . . . . . . . . . . 11

3.1.1 The Hamiltonian of the composite system . . . . . . . . . 11

3.1.2 Phenomenological modelling. . . . . . . . . . . . 13

3.1.3 Ohmic and frequency-dependent damping . . . . . . . 15

3.2 Microscopic models . . . . . . . . . . . . . . . 17

3.2.1 Optical and acoustic polarons . . . . . . . . . . . . 17

3.2.2 Interaction with fermions in the normal and superconducting state . . . . . . . . . . . . . . . . 19

3.2.3 Superconducting tunnel junction ........... 22

4 Imaginary-time path integrals $\mathbf{2 3}$

4.1 Equilibrium density matrix of the open system . . . . . . . . 25

4.1.1 Linear response model of Caldeira and Leggett . . . . . . . 26

4.1 .2 Optical polarons . . . . . . . . . . . . . 30

4.1.3 Acoustic polarons, interstitials, and defects . . . . . . . 32

4.1.4 Heavy particle in normally conducting and superconducting environment. . . . . . . . . . . . . . 33

4.1.5 Effective action of a Josephson junction . . . . . . . . 42

4.2 Partition function of the open system $\ldots \ldots \ldots \ldots$

4.2 .1 Semiclassical limit . . . . . . . . . . . . . . . 50

4.2.2 The effective classical potential . . . . . . . . 53

4.2 .3 Variational method . . . . . . . . . . . . . 55 
5 Real-time path integrals and dynamics 58

5.1 Feynman-Vernon method for a product initial state . . . . . . 60

5.2 General initial states and preparation function $\ldots \ldots \ldots 65$

5.3 Complex-time path integral for the propagating function $\ldots \ldots 66$

5.4 Real-time path integral for the propagating function . . . . . . 70

5.4 .1 Extremal paths . . . . . . . . . . . . . . 74

5.4 .2 Classical limit . . . . . . . . . . . . . . . 75

5.4.3 Semiclassical limit: quasiclassical Langevin equation . . . . 75

5.5 Brief summary and outlook . . . . . . . . . . . . 78

II SIMPLE DISSIPATIVE QUANTUM SYSTEMS

6 Damped harmonic oscillator $\quad 79$

6.1 Stochastic modelling . . . . . . . . . . . . . . . . . 79

6.2 Fluctuation-dissipation theorem . . . . . . . . . . . 81

6.3 The coordinate autocorrelation function $\ldots \ldots \ldots \ldots \ldots$

6.4 Ohmic friction . . . . . . . . . . . . . . . 85

6.5 Mean square of coordinate and momentum . . . . . . . . 87

6.6 The equilibrium density matrix $\ldots \ldots \ldots \ldots \ldots$

7 Quantum Brownian motion 93

7.1 Spectral density and damping coefficient . . . . . . . . . 93

7.2 Displacement correlations and response function $\ldots \ldots \ldots$. . . 95

7.3 Ohmic damping . . . . . . . . . . . . . . . . . . . 97

7.4 Frequency-dependent damping . . . . . . . . . . . . 99

III QUANTUM-STATISTICAL DECAY 103

8 Introduction to the problem 103

8.1 Formulation of the problem . . . . . . . . . . . . . . . . 104

8.2 Classical rate theory . . . . . . . . . . . . . . . 105

8.3 Quantum transition state theory . . . . . . . . . . . . 107

8.4 Free energy of a dissipative metastable system . . . . . . . . . . 112

9 The crossover temperature $T_{0} \quad 113$

10 Thermally activated decay 115

10.1 Imaginary part of the free energy $\ldots \ldots \ldots \ldots \ldots \ldots \ldots$

10.2 Rate formula and classical limit . . . . . . . . . . . . . 117

10.3 Quantum corrections . . . . . . . . . . . . . . . . . . . . 119

10.4 Multidimensional quantum transition state theory $\ldots \ldots \ldots 120$ 
11 The crossover region $\quad 126$

11.1 Beyond steepest descent for $T>T_{0} \ldots \ldots \ldots \ldots \ldots \ldots$

11.2 Beyond steepest descent for $T<T_{0} \ldots \ldots \ldots \ldots \ldots \ldots$

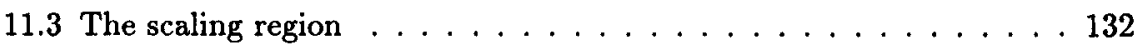

12 Dissipative quantum tunneling 134

12.1 The quantum rate formula . . . . . . . . . . . . . . . 134

12.2 Thermal enhancement of macroscopic quantum tunneling . . . . 137

12.3 Concluding remarks $\ldots \ldots \ldots \ldots . \ldots \ldots$

IV THE DISSIPATIVE TWO-STATE SYSTEM

13 Introduction $\quad 141$

14 Thermodynamics $\quad 144$

14.1 Partition function and specific heat $\ldots \ldots \ldots \ldots \ldots . \ldots 144$

14.1.1 Exact formal expression for the partition function . . . . 144

14.1.2 The limit of high temperatures . . . . . . . . . 147

14.1.3 Noninteracting-kink-pair approximation . . . . . . . . 148

14.1.4 The limit of weak coupling . . . . . . . . . . . . . 149

14.1.5 The Ohmic case . . . . . . . . . . . . . 150

14.1.6 The super-Ohmic case . . . . . . . . . . . . 152

14.2 Spin-boson model with adiabatic bath . . . . . . . . . 153

14.3 Incoherent tunneling in a double well . . . . . . . . . . . . . 154

14.3.1 Imaginary part of the free energy and quantum rate formula 154

14.3.2 Results for Ohmic dissipation . . . . . . . . . . 155

14.4 Relation to the Kondo problem . . . . . . . . . . . . 156

14.4.1 Anisotropic Kondo model . . . . . . . . . . . . . . 157

14.4.2 Resonant level model . . . . . . . . . . . . . . . . . . 159

15 Two-state dynamics $\quad 159$

15.1 Initial preparation . . . . . . . . . . . . . . 159

15.2 Exact formal expressions for the system dynamics $\ldots \ldots \ldots 162$

15.3 The noninteracting-blip approximation . . . . . . . . 166

15.3.1 Ohmic case . . . . . . . . . . . . . . 168

15.4 Biased system in the weak-coupling limit . . . . . . . . . 172

15.5 Ohmic dissipation with the special value $K=1 / 2 \ldots \ldots \ldots$. . . . 174

15.6 Long-time behavior of $P(t)$ and $C(t)$ at zero temperature . . . . 177

15.7 Thermodynamic properties . . . . . . . . . . . . . 181 
16 Dynamical Simulation 183

16.1 Stationary Phase Monte Carlo method . . . . . . . . . . . . . 184

16.2 Discretized path summation formula . . . . . . . . . . . 186

16.3 SPMC algorithm . . . . . . . . . . . . . . . . 190

16.4 Simulation results $\ldots \ldots \ldots \ldots \ldots \ldots \ldots$

16.4.1 Ohmic dissipation . . . . . . . . . . . 193

16.4 .2 Non-Ohmic dissipation . . . . . . . . . . . . . . 194

16.4.3 Brief summary . . . . . . . . . . . . . . 196

V THE DISSIPATIVE MULTI-STATE SYSTEM 198

$\begin{array}{ll}17 \text { Introduction } & 198\end{array}$

18 General formalism 200

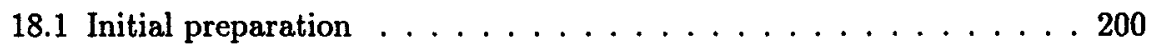

18.2 Exact formal expressions for the system dynamics $\ldots \ldots \ldots 203$

19 Dynamics at long times 208

19.1 Expressions for transport coefficients . . . . . . . . . . . 208

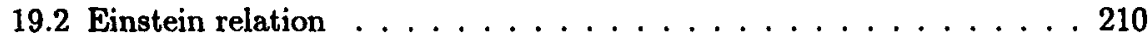

19.3 The Ohmic case . . . . . . . . . . . . . . . . . . . . . 211

19.3.1 Quantum diffusion for high temperatures and/or strong damping . . . . . . . . . . . . . . . 211

19.3.2 Mobility . . . . . . . . . . . . . . . . 212

19.3.3 Variance of the position . . . . . . . . . . . . . 214

19.3.4 Linear response . . . . . . . . . . . . . . . . 215

19.3.5 The case $K=1 / 2 \ldots \ldots \ldots \ldots \ldots \ldots \ldots$

$\begin{array}{ll}\text { Bibliography } & 221\end{array}$

Index $\quad 233$ 


\section{GUANTUM DISSIPATIVE SYSTEMS}

हี

to

记

옹 